\title{
A note on the solution of the electro-elastic boundary-value problem for rank-two laminates at finite strains
}

\author{
Valentina Volpini • Lorenzo Bardella $(\mathbb{D} \cdot$ Massimiliano Gei $(\mathbb{D}$
}

Received: 8 October 2018/Accepted: 23 March 2019/Published online: 9 April 2019

(C) The Author(s) 2019

\begin{abstract}
Recent research has shown that hierarchical laminated composites can be profitably employed to improve the actuation performance of electricallyactivated soft dielectric transducers. This note focuses on two types of rank-two layouts composed of ideal dielectric phases which follow nonlinear hyper-elastic mechanical behaviour and aims at providing a simplified set of solving equations for voltage-controlled actuation. We obtain such equations by analytical manipulations allowing to partly uncouple the set of equations usually employed within this theoretical framework. By focusing on neo-Hookean hyperelasticity, we validate the proposed methodology with the results available in literature for one layout. For the other layout, we obtain new configurations by maximising the axial stretch. In both cases, we study the sensitivity of the optimal actuation stretch to changes of the parameters characterising the rank-two mesoand micro-structures. In average, the computational time required to reach a convergent solution with the new methodology is one order of magnitude lower
\end{abstract}

V. Volpini · L. Bardella

Department of Civil, Environmental, Architectural Engineering and Mathematics, University of Brescia, Via Branze 43, 25123 Brescia, Italy

M. Gei ( ()

School of Engineering, Cardiff University, The Parade, Cardiff CF24 3AA, UK

e-mail: geim@cardiff.ac.uk than that necessary to solve the whole set of nonlinear coupled equations.

Keywords Dielectric elastomers - Composite materials $\cdot$ Smart materials $\cdot$ Finite strain . Electromechanical actuation

\section{Introduction}

The use of hierarchical composites is a possible solution to the quest for the enhancement of actuation performance of soft electroactive materials. The effectiveness of nested layered electro-elastic composites in achieving this goal has been made evident in a set of contributions [1-5] where emerging shortcomings, mainly associated with amplification of local electric fields and the risk of onset of damage at internal interfaces, have been also highlighted.

Generic dielectric rank- $N$ laminates, where $N$ is the hierarchical order, subjected to a given electric field are thoroughly analysed by Tian et al. [2] in the linear elastic regime. These authors demonstrate that the gain in actuation strain for a traction-free specimen obtained at an increasing contrast in the electromechanical properties of phases almost follows an exponential law. Gei and Mutasa [5] extend these findings by investigating optimal layouts for rank-two composites in a fully nonlinear electro-elastic framework for soft elastomers, governed by a neo-Hookean 
constitutive law and ideal dielectric behaviour. For the studied configurations, they show that accounting for nonlinearities leads to actuation strains that are up to $18 \%$ higher than those predicted at small strains and that the optimum arrangement of phases strongly depends on the maximum electric field for which the transducer is designed. By resorting to numerical simulations, Rudykh et al. [3] find a ten-fold improvement of the electro-mechanical coupling for a prototype rank-two laminate obtained by reinforcing an acrylic elastomer matrix with polyaniline. All these works completely neglect macro- and micro-scopic instabilities, that could be studied with the methods presented in [6-13].

This note deals with the solution of the actuation problem of rank-two laminated thin-films subjected to an electric potential difference across perfectly compliant electrodes placed on their sides [14]. ${ }^{1}$ As detailed in Sect. 2, the actuation response of an electro-elastic rank-two composite can be formally computed by coupling two rank-one problems. By referring to the notation introduced in Fig. 1, those two problems are: i) the microscopic one within the core and ii) the mesoscopic task involving core and shell.

For a material behaviour similar to that assumed in [5], as specified in Sect. 3, Spinelli and Lopez-Pamies [12] have shown that an explicit form of the free energy density can be directly formulated for an electro-elastic rank-one material. Therefore, the actuation performance here of interest can be estimated by just solving the mesoscopic rank-one problem through the coupling between the external boundary prescriptions expressed in terms of macroscopic quantities and the electro-mechanical continuity conditions at the interface characterising the laminate meso-structure.

The goal of this investigation, pursued in Sect. 4, is to analytically simplify at lowest terms the set of nonlinear coupled equations to easily solve the electro-elastic rank-two problem for voltage-controlled actuation. To this purpose, we consider two different layouts, displayed in Fig. 1 and, here and henceforth, referred to as 'Tree a' and 'Tree b', under the plane-strain assumption. However, as shown in Appendix, this methodology can be also easily

\footnotetext{
${ }^{1}$ It is here worth mentioning that an alternative actuation process is that where charges are directly deposited onto the actuator sides [15].
}

extended to the more general boundary-value problem in which the constraint imposed along the out-of-plane direction is released.

We numerically study the 'Tree a' and 'Tree b' microstructures in Sect. 5. Specifically, we first adopt the 'Tree a' configuration to validate the methodology developed in this work by comparison of the actuation stretches against literature results [2,5]. With regard to the 'Tree b' configuration, we provide novel data for microstructure arrangements able to optimise the actuation stretch. Moreover, for both layouts, we study the sensitivity of the maximum actuation stretch to changes of the parameters characterising the ranktwo meso- and micro-structures.

We finally assess the computational efficiency of the proposed reduced system of solving equations by comparing its performance with that of the fully coupled nonlinear equations usually employed in literature.

\section{Homogenisation of a rank-two dielectric composite actuator}

The rank-two laminate is constructed by properly embedding a reinforcement phase $a$ in a softer matrix $b$. In particular, such a heterogeneous transducer can be designed in two different ways [2], according to the layouts sketched in Fig. 1 that are independent of direction $x_{3}$. In the first case, referred to as 'Tree a', the device is obtained by layering a core rank-one composite (whose relevant variables are henceforth labelled with 'R1') with layers of the soft material $b$ acting as a shell. In our terminology, the shell is a purely homogeneous material and its quantities are labelled with 'sh'. In the second case, referred to as 'Tree b', the rank-one core is sandwiched between layers of the stiffer material $a$, here playing the role of the shell.

We assume separation of length-scales such that each rank can be homogenised independently. More specifically, the local fields within the rank-one composite are microscopic fields, whereas, at a much larger scale, the mesoscopic fields are the local fields for the rank-two composite, in which the rank-one core is modelled as a homogeneous phase.

Within this picture, at the mesoscopic level, $c^{\mathrm{R} 1}$ and $c^{\mathrm{sh}}=1-c^{\mathrm{R} 1}$ denote the volume fractions of core and 
Fig. 1 Schematics of the reference configuration of the studied rank-two layered dielectric actuator subjected to voltage difference $\Delta \phi$ applied across flexible electrodes. The close-up views highlight the rank-one core and the shell composed of a the soft matrix $b$ ('Tree a'), in light gray, or $\mathbf{b}$ the reinforcement $a$ ('Tree b'), in dark gray. The initial thickness of the actuator is $h^{0}$. According to the positive direction of the out-of-plane axis $x_{3}$, angles $\theta_{\mathrm{R} 1}$ and $\theta_{\mathrm{sh}}$ are positive if anticlockwise

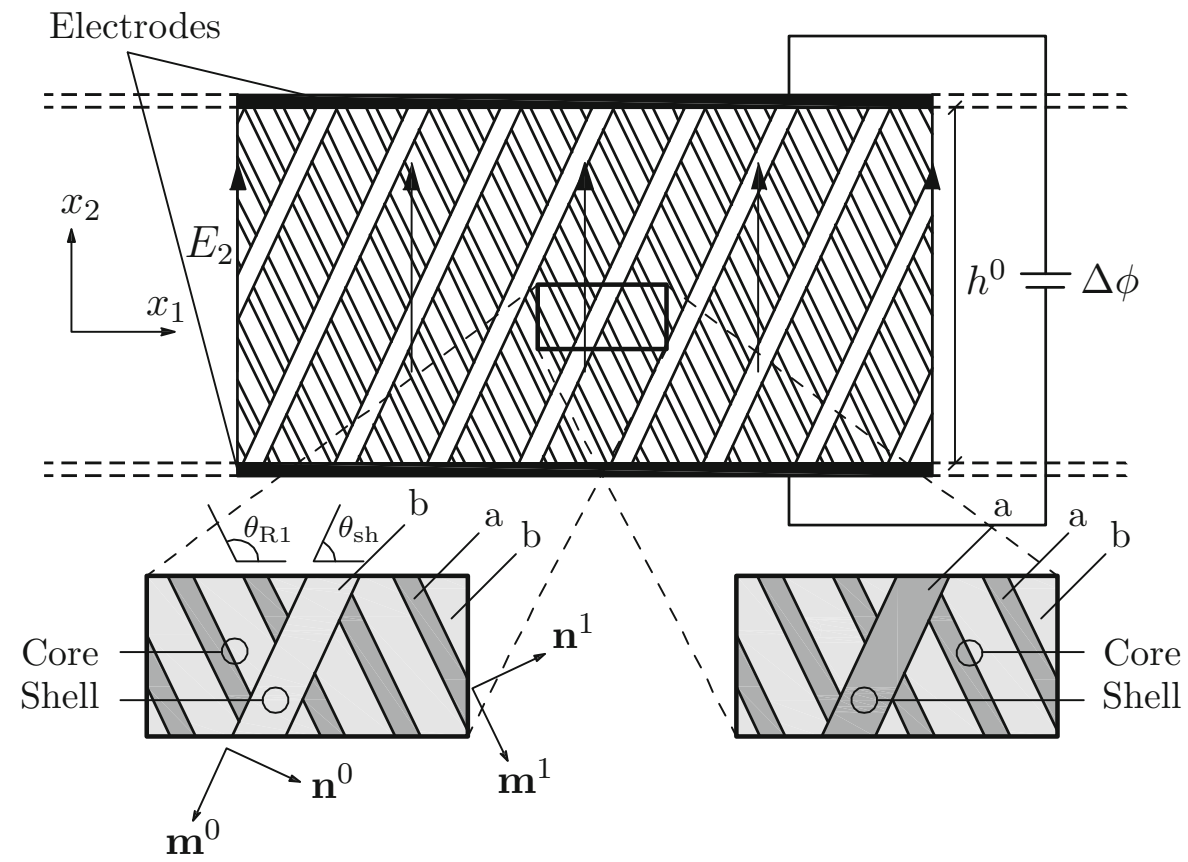

(a) shell, respectively, the former reading $c^{\mathrm{R} 1}=c_{a}^{\mathrm{R} 1}+c_{b}^{\mathrm{R} 1}$. At the microscopic level, the homogenisation of the rank-one core requires the use of the volume fractions $c_{a}^{\mathrm{R} 1} / c^{\mathrm{R} 1}$ and $c_{b}^{\mathrm{R} 1} / c^{\mathrm{R} 1}$ for the two phases $a$ and $b$, respectively. Finally, $c^{a}$ and $c^{b}$ are the overall volume fractions of the two homogeneous materials entering the whole composite, such that $c^{a}+c^{b}=1$. In particular, it results:

$c^{a}=c_{a}^{\mathrm{R} 1}$ and $c^{b}=c_{b}^{\mathrm{R} 1}+c^{\mathrm{sh}} \quad$ in 'Tree a' configuration,

while

$c^{a}=c_{a}^{\mathrm{R} 1}+c^{\mathrm{sh}}$ and $c^{b}=c_{b}^{\mathrm{R} 1} \quad$ in 'Tree b' configuration.

The interfaces between phases $a$ and $b$ in the core are henceforth denoted as microscopic, whereas the mesoscopic interfaces are those separating shell and rank-one phases in the rank-two composite. The normal and tangential unit vectors defining the microscopic and mesoscopic interfaces belong to planes $x_{3}=$ const and are indicated as $\left(\mathbf{n}^{1}, \mathbf{m}^{1}\right)$ and $\left(\mathbf{n}^{0}, \mathbf{m}^{0}\right)$, respectively (see Fig. 1); we express their Cartesian components in terms of the interfaces' angles with respect to the axis $x_{1}$, denoted as $\theta_{\mathrm{R} 1}$ and $\theta_{\text {sh }}$, respectively. These angles are positive if anticlockwise, as illustrated in Fig. 1. In both 'Tree a' and
'Tree b' configurations, the normal vector $\mathbf{n}^{1}$ always points from the stiffer phase $a$ towards the softer phase $b$, while the normal vector $\mathbf{n}^{0}$ points from the rank-one core towards the shell.

In this work, the relevant mesoscopic fields, assumed to be spatially uniform, are the deformation gradient $\mathbf{F}_{k}(k=\mathrm{sh}, \mathrm{R} 1)$ and the nominal electric field $\mathbf{E}_{k}$, along with their work-conjugate quantities, that are the first Piola-Kirchhoff stress $\mathbf{S}_{k}$ and the nominal electric displacement $\mathbf{D}_{k}$. The analogous macroscopic electro-mechanical quantities governing the overall response of the actuator are indicated as F, E, S, D.

Under voltage-controlled actuation in plane-strain conditions, we assign the through-the-thickness macroscopic nominal electric field component

$E_{2}=\frac{\Delta \phi}{h^{0}}$,

in which $\Delta \phi$ is the electric potential jump applied across the electrodes and $h^{0}$ is the initial laminate thickness. Additionally, we impose

$E_{1}=0$,

which is consistent with disregarding edge effects. This is coupled with macroscopic traction-free boundary conditions: 


$$
\begin{aligned}
& S_{22}=0, \\
& S_{12}=0, \\
& S_{11}=0,
\end{aligned}
$$

the latter allowing free stretch $\lambda$ along the $x_{1}$ direction (see Fig. 1). Under these conditions, the deformation gradient assumes the form

$$
\mathbf{F}=\left[\begin{array}{ccc}
\lambda & \xi / \lambda & 0 \\
0 & 1 / \lambda & 0 \\
0 & 0 & 1
\end{array}\right],
$$

in which the term $\xi$ represents the amount of shear associated with the actual shear angle $\gamma=\arctan \xi$.

We remark that the methodology developed in this investigation can be equally applied when the deformation along $x_{3}$ is unconstrained. Hence, in Appendix, we indicate how to solve this dual boundary-value problem by utilising exactly the same equations as those obtained in the following. More importantly, on the basis of numerical investigations, in Appendix, we draw conclusions on the appropriateness of the planestrain assumption.

Within the proposed two-scale framework, we obtain the overall actuation response through homogenisation of the mesoscopic level by following the same technique proposed in [4] for the rank-one composite. The main novelty in our study of the ranktwo composite consists of using for the rank-one core phase the free energy density obtained by Spinelli and Lopez-Pamies [12], which is different from that characterising the shell. Conversely, in the computational and analytical investigations of Gei et al. [4] and Spinelli and Lopez-Pamies [12], the two phases constituting the rank-one composite therein studied have the same form of free energy density. In other words, the effective energy density of Spinelli and Lopez-Pamies [12] is in our context the analytical result of the microscopic rank-one homogenisation into a homogeneous mesoscopic phase. We remark that at both levels the homogenisation takes advantage of the condition that the interface normal (either $\mathbf{n}^{1}$ or $\mathbf{n}^{0}$ ) is spatially uniform.

The homogenisation technique consists of coupling of information on the continuity of electro-mechanical variables at the mesoscopic interface with the definition of macroscopic quantities as weighed averages of mesoscopic fields [16-18].

On the one hand, continuity at the mesoscopic interface is expressed by [19]

$$
\begin{aligned}
& \left(\mathbf{F}_{\mathrm{sh}}-\mathbf{F}_{\mathrm{R} 1}\right) \mathbf{m}^{0}=\mathbf{0}, \\
& \left(\mathbf{S}_{\mathrm{sh}}-\mathbf{S}_{\mathrm{R} 1}\right) \mathbf{n}^{0}=\mathbf{0}, \\
& \left(\mathbf{E}_{\mathrm{sh}}-\mathbf{E}_{\mathrm{R} 1}\right) \cdot \mathbf{m}^{0}=0, \\
& \left(\mathbf{D}_{\mathrm{sh}}-\mathbf{D}_{\mathrm{R} 1}\right) \cdot \mathbf{n}^{0}=0 .
\end{aligned}
$$

where Eq. (2c) is obtained from the general relation $\mathbf{n}^{0} \times\left(\mathbf{E}_{\mathrm{sh}}-\mathbf{E}_{\mathrm{R} 1}\right)=\mathbf{0}$ particularised to the case here of interest, in which both $\mathbf{n}^{0}$ and the electric field vector have vanishing component along the $x_{3}$ direction (see Fig. 1). Moreover, here and henceforth, $\times$ and . denote, respectively, the vector and inner products.

On the other hand, the macroscopic fields read

$$
\begin{aligned}
& \mathbf{F}=c^{\mathrm{sh}} \mathbf{F}_{\mathrm{sh}}+c^{\mathrm{R} 1} \mathbf{F}_{\mathrm{R} 1}, \\
& \mathbf{E}=c^{\mathrm{sh}} \mathbf{E}_{\mathrm{sh}}+c^{\mathrm{R} 1} \mathbf{E}_{\mathrm{R} 1}, \\
& \mathbf{D}=c^{\mathrm{sh}} \mathbf{D}_{\mathrm{sh}}+c^{\mathrm{R} 1} \mathbf{D}_{\mathrm{R} 1},
\end{aligned}
$$

such that fulfillment of $(2 a),(2 c),(2 d)$ requires the following forms of the mesoscopic fields in terms of the scalar coefficients $\alpha, \beta$, and $\bar{\beta}$ [11]

$$
\begin{aligned}
& \mathbf{F}_{\mathrm{sh}}=\mathbf{F}\left(\mathbf{I}+\alpha c^{\mathrm{R} 1} \mathbf{m}^{0} \otimes \mathbf{n}^{0}\right), \\
& \mathbf{F}_{\mathrm{R} 1}=\mathbf{F}\left(\mathbf{I}-\alpha c^{\mathrm{sh}} \mathbf{m}^{0} \otimes \mathbf{n}^{0}\right),
\end{aligned}
$$

where $\otimes$ denotes the outer product,

$$
\begin{aligned}
& \mathbf{E}_{\mathrm{sh}}=\mathbf{E}+c^{\mathrm{R} 1} \beta \mathbf{n}^{0}, \\
& \mathbf{E}_{\mathrm{R} 1}=\mathbf{E}-c^{\mathrm{sh}} \beta \mathbf{n}^{0}, \\
& \mathbf{D}_{\mathrm{sh}}=\mathbf{D}+c^{\mathrm{R} 1} \bar{\beta} \mathbf{m}^{0}, \\
& \mathbf{D}_{\mathrm{R} 1}=\mathbf{D}-c^{\mathrm{sh}} \bar{\beta} \mathbf{m}^{0} .
\end{aligned}
$$

The coefficient $\alpha$ is a dimensionless parameter, whereas $\beta$ and $\bar{\beta}$ have the dimensions of an electric field and an electric displacement, respectively. Parameters $\alpha, \beta$, and $\bar{\beta}$ are determined by imposing further electro-mechanical conditions, involving the 
mesoscopic constitutive laws, that can be expressed in terms of the free energy densities $W_{k}\left(\mathbf{F}_{k}, \mathbf{E}_{k}\right)$ [20]

$$
\begin{aligned}
& \mathbf{S}_{k}=\frac{\partial W_{k}}{\partial \mathbf{F}_{k}}-p_{k} \mathbf{F}_{k}^{-T}, \\
& \mathbf{D}_{k}=-\frac{\partial W_{k}}{\partial \mathbf{E}_{k}} .
\end{aligned}
$$

As an important issue to address in this investigation, in Eq. (7a) we have assumed materials constrained to undergo isochoric deformation, such that the stress depends on the Lagrangian multiplier $p_{k}$, to be determined on each phase.

At the macroscopic level, the effective electroelastic free energy density is the sum of the weighed mesoscopic energies, namely

$$
\begin{aligned}
W(\mathbf{F}, \mathbf{E}) & =c^{\mathrm{sh}} W_{\mathrm{sh}}\left(\mathbf{F}_{\mathrm{sh}}, \mathbf{E}_{\mathrm{sh}}\right)+c^{\mathrm{R} 1} W_{\mathrm{R} 1}\left(\mathbf{F}_{\mathrm{R} 1}, \mathbf{E}_{\mathrm{R} 1}\right) \\
& =c^{\mathrm{sh}} \tilde{W}_{\mathrm{sh}}(\alpha, \beta)+c^{\mathrm{R} 1} \tilde{W}_{\mathrm{R} 1}(\alpha, \beta),
\end{aligned}
$$

where, by resorting to Eqs. (4) and (5), we have expressed the mesoscopic energies as functions of $\alpha$ and $\beta$. More precisely, the function $W$ in Eq. (8) is the effective energy only for $\alpha$ and $\beta$ fulfilling the required conditions of the set boundary-value problem. We note that, in the voltage-controlled problem here of concern, the effective response of the rank-two composite is completely determined by the parameters $\alpha$ and $\beta$, while $\bar{\beta}$ would directly enter the effective response in the dual charge-controlled problem.

Analogously to (7), the macroscopic constitutive equations read $[6,10]$

$$
\begin{aligned}
& \mathbf{S}=\frac{\partial W}{\partial \mathbf{F}}-p \mathbf{F}^{-T}, \\
& \mathbf{D}=-\frac{\partial W}{\partial \mathbf{E}}
\end{aligned}
$$

where

$p=c^{\mathrm{sh}} p_{\mathrm{sh}}+c^{\mathrm{R} 1} p_{\mathrm{R} 1}$.

Now, we focus on some general results, inherent to our homogenisation framework, which can be conveniently employed in the computations, as explained in detail in Sects. 4 and 5.

First, by combining Eqs. (9b) and (8), we obtain

$$
\begin{aligned}
\mathbf{D}= & -\left[c^{\mathrm{sh}}\left(\frac{\partial W_{\mathrm{sh}}}{\partial \mathbf{F}_{\mathrm{sh}}} \frac{\mathrm{d} \mathbf{F}_{\mathrm{sh}}}{\mathrm{d} \mathbf{E}}+\frac{\partial W_{\mathrm{sh}}}{\partial \mathbf{E}_{\mathrm{sh}}} \frac{\mathrm{d} \mathbf{E}_{\mathrm{sh}}}{\mathrm{d} \mathbf{E}}\right)\right. \\
& \left.+c^{\mathrm{R} 1}\left(\frac{\partial W_{\mathrm{R} 1}}{\partial \mathbf{F}_{\mathrm{R} 1}} \frac{\mathrm{d} \mathbf{F}_{\mathrm{R} 1}}{\mathrm{~d} \mathbf{E}}+\frac{\partial W_{\mathrm{R} 1}}{\partial \mathbf{E}_{\mathrm{R} 1}} \frac{\mathrm{d} \mathbf{E}_{\mathrm{R} 1}}{\mathrm{~d} \mathbf{E}}\right)\right],
\end{aligned}
$$

in which our notation for the chian rule implies $[(\partial W / \partial \mathbf{F})(\mathrm{d} \mathbf{F} / \mathrm{d} \mathbf{E})]_{k} \equiv\left(\partial W / \partial F_{i j}\right)\left(\mathrm{d} F_{i j} / \mathrm{d} E_{k}\right) \quad$ with $i, j, k$ indices with respect to a Cartesian system.

By accounting, in Eq. (10), for the dependence of the mesoscopic fields on the macroscopic quantities $\mathbf{F}(\mathbf{E}), \alpha(\mathbf{E}), \beta(\mathbf{E})$ through Eqs. (4) and (5), we find out that the sums of the contributions multiplying $\mathrm{d} \alpha / \mathrm{d} \mathbf{E}$ and $\mathrm{d} \beta / \mathrm{d} \mathbf{E}$ turn out into the left-hand sides of continuity conditions (2b) and (2d), respectively. Similarly, two contributions involving $\mathrm{d} \mathbf{F} / \mathrm{d} \mathbf{E}$ multiply terms that allow one to single-out both the lefthand side of condition ( $2 b)$ and the macroscopic stress S. Finally, the product $\left(\mathbf{F}^{-T} \mathrm{~d} \mathbf{F} / \mathrm{d} \mathbf{E}\right)_{k} \equiv F_{j i}^{-1} \mathrm{~d} F_{i j} / \mathrm{d} E_{k}$ represents the variation of $\operatorname{det} \mathbf{F}$, to be neglected in the case of isochoric deformation. Hence, all these terms vanish. Because of this, it results that, in order to correctly evaluate Eq. (3c) through Eq. (10), we may completely disregard the dependence of $\alpha, \beta$, and $\mathbf{F}$ on E.

Analogously, by combining Eqs. (9a) and (8), we obtain

$$
\begin{aligned}
\mathbf{S}= & c^{\mathrm{sh}}\left(\frac{\partial W_{\mathrm{sh}}}{\partial \mathbf{F}_{\mathrm{sh}}} \frac{\mathrm{d} \mathbf{F}_{\mathrm{sh}}}{\mathrm{d} \mathbf{F}}+\frac{\partial W_{\mathrm{sh}}}{\partial \mathbf{E}_{\mathrm{sh}}} \frac{\mathrm{d} \mathbf{E}_{\mathrm{sh}}}{\mathrm{d} \mathbf{F}}\right) \\
& +c^{\mathrm{R} 1}\left(\frac{\partial W_{\mathrm{R} 1}}{\partial \mathbf{F}_{\mathrm{R} 1}} \frac{\mathrm{d} \mathbf{F}_{\mathrm{R} 1}}{\mathrm{~d} \mathbf{F}}+\frac{\partial W_{\mathrm{R} 1}}{\partial \mathbf{E}_{\mathrm{R} 1}} \frac{\mathrm{d} \mathbf{E}_{\mathrm{R} 1}}{\mathrm{~d} \mathbf{F}}\right)-p \mathbf{F}^{-T} .
\end{aligned}
$$

Calculations similar to those concerned with $\mathbf{D}$ lead to the result that the relation

$$
c^{\mathrm{sh}} \mathbf{S}_{\mathrm{sh}}+c^{\mathrm{R} 1} \mathbf{S}_{\mathrm{R} 1}=\mathbf{S} \equiv \mathbf{0}
$$

is correctly evaluated through Eq. (11) even by totally neglecting the derivatives $\mathrm{d} \alpha / \mathrm{d} \mathbf{F}$ and $\mathrm{d} \beta / \mathrm{d} \mathbf{F}$, as they multiply contributions that cancel out.

These observations are particularly useful in solving our problem as they allow us to end up with an algebraic system involving, among the unknowns, only a single Lagrangian multiplier $p$. Given the nonlinearity of the system providing the solution, the analytical development proposed in this work leads to a relevant computational advantage with respect to an approach directly implementing all the governing 
equations, where both mesoscopic pressures enter the system unknowns.

Moreover, within our homogenisation procedure, by combining Eqs. (11) and (1a), we can analytically obtain $p$ as a function of $\alpha, \beta, \lambda$, and $\xi$, thus further reducing the dimension of the solving nonlinear system.

We finally note that in other analogous voltagecontrolled problems, such as that with imposed vanishing shear deformation, $F_{12}=0$, and $S_{12}$ to be determined among the macroscopic unknowns, the foregoing observations about relations (10) and (11) still hold.

In the next section, we specify the mesoscopic free energy densities characterising the rank-two laminate.

\section{The mesoscopic constitutive prescriptions}

We assume hyper-electro-elastic material behaviour for both the matrix and the reinforcement, all constrained to undergo isochoric deformation governed by an extended neo-Hookean strain-energy function with embedded ideal dielectricity. This choice requires the introduction of two material parameters for each phase, namely, the shear moduli $\mu_{a}$ and $\mu_{b}$ and the dielectric permittivities $\epsilon_{a}$ and $\epsilon_{b}$. Hence, the material constants of the shell phase are $\mu_{\mathrm{sh}}=\mu_{b}$ and $\epsilon_{\mathrm{sh}}=\epsilon_{b}$ in 'Tree a' configuration, while $\mu_{\mathrm{sh}}=\mu_{a}$ and $\epsilon_{\mathrm{sh}}=\epsilon_{a}$ for 'Tree b' microstructure (see Fig. 1).

In the case here of interest, where the deformation is voltage-driven, we choose the nominal electric field as the primal electric variable. Moreover, among different possibilities inherent to the finite deformation framework, we adopt the electrostatic contribution to the energy to be dependent on the non-standard invariant $\left|\mathbf{F}_{k}^{-T} \mathbf{E}_{k}\right|$, with $|\mathbf{E}| \equiv \sqrt{\mathbf{E} \cdot \mathbf{E}}$ denoting the modulus.

Therefore, the free energy density of the shell may be expressed as

$W_{\mathrm{sh}}\left(\mathbf{F}_{\mathrm{sh}}, \mathbf{E}_{\mathrm{sh}}\right)=\frac{\mu_{\mathrm{sh}}}{2}\left(\operatorname{tr} \mathbf{C}_{\mathrm{sh}}-3\right)-\frac{\epsilon_{\mathrm{sh}}}{2}\left|\mathbf{F}_{\mathrm{sh}}^{-T} \mathbf{E}_{\mathrm{sh}}\right|^{2}$,

where $\operatorname{tr} \mathbf{C}_{k}$ is the trace of the right Cauchy-Green tensor
$\mathbf{C}_{k}=\mathbf{F}_{k}^{T} \mathbf{F}_{k}$.

For the rank-one mesoscopic laminate constituted by phases governed by the potential (12), we use the homogenised energy potential analytically obtained by Spinelli and Lopez-Pamies [12]

$$
\begin{aligned}
W_{\mathrm{R} 1}\left(\mathbf{F}_{\mathrm{R} 1}, \mathbf{E}_{\mathrm{R} 1}\right)= & \frac{\mu_{V}}{2}\left(\operatorname{tr} \mathbf{C}_{\mathrm{R} 1}-3\right) \\
& -\frac{\mu_{V}-\mu_{R}}{2}\left(\left|\mathbf{F}_{\mathrm{R} 1} \mathbf{n}^{1}\right|^{2}-\left|\mathbf{F}_{\mathrm{R} 1}^{-T} \mathbf{n}^{1}\right|^{-2}\right) \\
& -\frac{\epsilon_{V}}{2}\left|\mathbf{F}_{\mathrm{R} 1}^{-T} \mathbf{E}_{\mathrm{R} 1}\right|^{2} \\
& +\frac{\epsilon_{V}-\epsilon_{R}}{2} \frac{\left[\mathbf{C}_{\mathrm{R} 1}^{-1} \cdot\left(\mathbf{E}_{\mathrm{R} 1} \otimes \mathbf{n}^{1}\right)\right]^{2}}{\left|\mathbf{F}_{\mathrm{R} 1}^{-T} \mathbf{n}^{1}\right|^{2}},
\end{aligned}
$$

which, in particular, displays a dependence on the nonstandard invariant $\mathbf{C}_{k}^{-1} \cdot\left(\mathbf{E}_{k} \otimes \mathbf{n}^{1}\right)$, along with the following mesoscopic material parameters accounting for the heterogeneity of the rank-one laminate:

$\mu_{V}=\frac{c_{a}^{\mathrm{R} 1}}{c_{\mathrm{R} 1}} \mu_{a}+\frac{c_{b}^{\mathrm{R} 1}}{c_{\mathrm{R} 1}} \mu_{b}, \quad \epsilon_{V}=\frac{c_{a}^{\mathrm{R} 1}}{c_{\mathrm{R} 1}} \epsilon_{a}+\frac{c_{b}^{\mathrm{R} 1}}{c_{\mathrm{R} 1}} \epsilon_{b}$,

and

$\mu_{R}=\left(\frac{c_{a}^{\mathrm{R} 1}}{c_{\mathrm{R} 1} \mu_{a}}+\frac{c_{b}^{\mathrm{R} 1}}{c_{\mathrm{R} 1} \mu_{b}}\right)^{-1}, \quad \epsilon_{R}=\left(\frac{c_{a}^{\mathrm{R} 1}}{c_{\mathrm{R} 1} \epsilon_{a}}+\frac{c_{b}^{\mathrm{R} 1}}{c_{\mathrm{R} 1} \epsilon_{b}}\right)^{-1}$.

Assuming $\epsilon_{R}=\epsilon_{V}$ and $\mu_{R}=\mu_{V}$ makes the rank-one laminate microscopically homogeneous and its free energy (13) of the same form as that for the shell in Eq. (12).

The constitutive equations (7a) and (7b) provide the following expressions for the mesoscopic stress and electric displacement

$$
\mathbf{S}_{\mathrm{sh}}=\mu_{\mathrm{sh}} \mathbf{F}_{\mathrm{sh}}-p_{\mathrm{sh}} \mathbf{F}_{\mathrm{sh}}^{-T}+\epsilon_{\mathrm{sh}} \mathbf{F}_{\mathrm{sh}}^{-T} \mathbf{E}_{\mathrm{sh}} \otimes \mathbf{C}_{\mathrm{sh}}^{-1} \mathbf{E}_{\mathrm{sh}}
$$




$$
\begin{aligned}
\mathbf{S}_{\mathrm{R} 1}= & \mu_{V} \mathbf{F}_{\mathrm{R} 1}-p_{\mathrm{R} 1} \mathbf{F}_{\mathrm{R} 1}^{-T}-\left(\mu_{V}-\mu_{R}\right) \mathbf{F}_{\mathrm{R} 1} \mathbf{n}^{1} \otimes \mathbf{n}^{1} \\
& +\epsilon_{V} \mathbf{F}_{\mathrm{R} 1}^{-T} \mathbf{E}_{\mathrm{R} 1} \otimes \mathbf{C}_{\mathrm{R} 1}^{-1} \mathbf{E}_{\mathrm{R} 1} \\
& +\frac{\mu_{V}-\mu_{R}+\left(\epsilon_{V}-\epsilon_{R}\right)\left[\mathbf{C}_{\mathrm{R} 1}^{-1} \cdot\left(\mathbf{E}_{\mathrm{R} 1} \otimes \mathbf{n}^{1}\right)\right]^{2}}{\left|\mathbf{F}_{\mathrm{R} 1}^{-T} \mathbf{n}^{1}\right|^{4}} \\
& \times \mathbf{F}_{\mathrm{R} 1}^{-T} \mathbf{n}^{1} \otimes \mathbf{C}_{\mathrm{R} 1}^{-1} \mathbf{n}^{1}-\frac{\left(\epsilon_{V}-\epsilon_{R}\right) \mathbf{C}_{\mathrm{R} 1}^{-1} \cdot\left(\mathbf{E}_{\mathrm{R} 1} \otimes \mathbf{n}^{1}\right)}{\left|\mathbf{F}_{\mathrm{R} 1}^{-T} \mathbf{n}^{1}\right|^{2}} \\
& \times\left(\mathbf{F}_{\mathrm{R} 1}^{-T} \mathbf{E}_{\mathrm{R} 1} \otimes \mathbf{C}_{\mathrm{R} 1}^{-1} \mathbf{n}^{1}+\mathbf{F}_{\mathrm{R} 1}^{-T} \mathbf{n}^{1} \otimes \mathbf{C}_{\mathrm{R} 1}^{-1} \mathbf{E}_{\mathrm{R} 1}\right), \\
\mathbf{D}_{\mathrm{sh}}= & \epsilon_{\mathrm{sh}} \mathbf{C}_{\mathrm{sh}}^{-1} \mathbf{E}_{\mathrm{sh}}, \\
\mathbf{D}_{\mathrm{R} 1}= & \epsilon_{V} \mathbf{C}_{\mathrm{R} 1}^{-1} \mathbf{E}_{\mathrm{R} 1}-\left(\epsilon_{V}-\epsilon_{R}\right) \frac{\mathbf{C}_{\mathrm{R} 1}^{-1} \cdot\left(\mathbf{E}_{\mathrm{R} 1} \otimes \mathbf{n}^{1}\right)}{\left|\mathbf{F}_{\mathrm{R} 1}^{-T} \mathbf{n}^{1}\right|^{2}} \mathbf{C}_{\mathrm{R} 1}^{-1} \mathbf{n}^{1} .
\end{aligned}
$$

Next, we develop a semi-analytical procedure to efficiently determine the effective behaviour of the rank-two hyper-electro-elastic laminates under investigation.

\section{The reduced system of solving equations for the semi-analytical rank-two homogenisation}

The procedure developed in this section focuses on analytical manipulations for the determination of the localisation parameters $\alpha$ and $\beta$ characterising the mesoscopic deformation gradient and nominal electric field through Eqs. (4) and (5), respectively.

Substitution of Eqs. (17) into Eq. (2d) yields

$$
\begin{aligned}
& \epsilon_{\mathrm{sh}} \mathbf{C}_{\mathrm{sh}}^{-1} \cdot\left(\mathbf{E}_{\mathrm{sh}} \otimes \mathbf{n}^{0}\right)-\epsilon_{V} \mathbf{C}_{\mathrm{R} 1}^{-1} \cdot\left(\mathbf{E}_{\mathrm{R} 1} \otimes \mathbf{n}^{0}\right) \\
& \quad+\left(\epsilon_{V}-\epsilon_{R}\right) \frac{\mathbf{C}_{\mathrm{R} 1}^{-1} \cdot\left(\mathbf{E}_{\mathrm{R} 1} \otimes \mathbf{n}^{1}\right)}{\left|\mathbf{F}_{\mathrm{R} 1}^{-T} \mathbf{n}^{1}\right|^{2}} \mathbf{C}_{\mathrm{R} 1}^{-1} \cdot\left(\mathbf{n}^{1} \otimes \mathbf{n}^{0}\right)=0,
\end{aligned}
$$

which can be expressed in terms of the macroscopic quantities $\mathbf{F}$ and $\mathbf{E}$ through Eqs. (4) and (5). Hence, Eq. (18) can be solved for $\beta$ as a function of the parameter $\alpha$, still unknown, as follows

$$
\begin{aligned}
\beta(\alpha)= & \left\{\left[\epsilon_{V}-\epsilon_{\mathrm{sh}}-\alpha c^{\mathrm{sh}} L(\alpha) \mathbf{m}^{0} \cdot \mathbf{n}^{1}\right] \mathbf{C}^{-1} \cdot\left(\mathbf{E} \otimes \mathbf{n}^{0}\right)\right. \\
& +\alpha \mathbf{E} \cdot \mathbf{m}^{0}\left[c^{\mathrm{R} 1} \epsilon_{\mathrm{sh}}+c^{\mathrm{sh}} \epsilon_{V}-\alpha\left(c^{\mathrm{sh}}\right)^{2} L(\alpha) \mathbf{m}^{0} \cdot \mathbf{n}^{1}\right] \\
& \times\left|\mathbf{F}^{-T} \mathbf{n}^{0}\right|^{2}-L(\alpha)\left[\mathbf{C}^{-1} \cdot\left(\mathbf{E} \otimes \mathbf{n}^{1}\right)\right. \\
& \left.\left.+\alpha c^{\mathrm{sh}} \mathbf{E} \cdot \mathbf{m}^{0} \mathbf{C}^{-1} \cdot\left(\mathbf{n}^{0} \otimes \mathbf{n}^{1}\right)\right]\right\} \\
& \times\left\{\left[c^{\mathrm{R} 1} \epsilon_{\mathrm{sh}}+c^{\mathrm{sh}} \epsilon_{V}-\alpha\left(c^{\mathrm{sh}}\right)^{2} L(\alpha) \mathbf{m}^{0} \cdot \mathbf{n}^{1}\right]\right. \\
& \left.\times\left|\mathbf{F}^{-T} \mathbf{n}^{0}\right|^{2}-c^{\mathrm{sh}} L(\alpha) \mathbf{C}^{-1} \cdot\left(\mathbf{n}^{0} \otimes \mathbf{n}^{1}\right)\right\}^{-1}
\end{aligned}
$$

where the auxiliary function $L(\alpha)$ reads

$$
L(\alpha)=\frac{\epsilon_{V}-\epsilon_{R}}{H(\alpha)}\left(\mathbf{C}^{-1} \cdot\left(\mathbf{n}^{0} \otimes \mathbf{n}^{1}\right)+\alpha c^{\mathrm{sh}} \mathbf{m}^{0} \cdot \mathbf{n}^{1}\left|\mathbf{F}^{-T} \mathbf{n}^{0}\right|^{2}\right),
$$

which is in turn dependent on $H(\alpha)$, defined as

$$
\begin{aligned}
H(\alpha)= & \left|\mathbf{F}^{-T} \mathbf{n}^{1}\right|^{2}+2 \alpha c^{\mathrm{sh}} \mathbf{m}^{0} \cdot \mathbf{n}^{1} \mathbf{C}^{-1} \cdot\left(\mathbf{n}^{0} \otimes \mathbf{n}^{1}\right) \\
& +\left(\alpha c^{\mathrm{sh}} \mathbf{m}^{0} \cdot \mathbf{n}^{1}\right)^{2}\left|\mathbf{F}^{-T} \mathbf{n}^{0}\right|^{2} .
\end{aligned}
$$

Analogously, substitution of Eqs. (16) into Eq. (2b) yields

$$
\begin{aligned}
& {\left[\mu_{\mathrm{sh}} \mathbf{F}_{\mathrm{sh}}-p_{\mathrm{sh}} \mathbf{F}_{\mathrm{sh}}^{-T}+\epsilon_{\mathrm{sh}} \mathbf{F}_{\mathrm{sh}}^{-T} \mathbf{E}_{\mathrm{sh}} \otimes \mathbf{C}_{\mathrm{sh}}^{-1} \mathbf{E}_{\mathrm{sh}}\right.} \\
& \quad-\mu_{V} \mathbf{F}_{\mathrm{R} 1}+p_{\mathrm{R} 1} \mathbf{F}_{\mathrm{R} 1}^{-T}+\left(\mu_{V}-\mu_{R}\right) \mathbf{F}_{\mathrm{R} 1} \mathbf{n}^{1} \otimes \mathbf{n}^{1} \\
& -\epsilon_{V} \mathbf{F}_{\mathrm{R} 1}^{-T} \mathbf{E}_{\mathrm{R} 1} \otimes \mathbf{C}_{\mathrm{R} 1}^{-1} \mathbf{E}_{\mathrm{R} 1} \\
& \quad-\frac{\mu_{V}-\mu_{R}+\left(\epsilon_{V}-\epsilon_{R}\right)\left[\mathbf{C}_{\mathrm{R} 1}^{-1} \cdot\left(\mathbf{E}_{\mathrm{R} 1} \otimes \mathbf{n}^{1}\right)\right]^{2}}{\left|\mathbf{F}_{\mathrm{R} 1}^{-T} \mathbf{n}^{1}\right|^{4}} \\
& \quad \times \mathbf{F}_{\mathrm{R} 1}^{-T} \mathbf{n}^{1} \otimes \mathbf{C}_{\mathrm{R} 1}^{-1} \mathbf{n}^{1}+\frac{\left(\epsilon_{V}-\epsilon_{R}\right) \mathbf{C}_{\mathrm{R} 1}^{-1} \cdot\left(\mathbf{E}_{\mathrm{R} 1} \otimes \mathbf{n}^{1}\right)}{\left|\mathbf{F}_{\mathrm{R} 1}^{-T} \mathbf{n}^{1}\right|^{2}} \\
& \left.\quad \times\left(\mathbf{F}_{\mathrm{R} 1}^{-T} \mathbf{E}_{\mathrm{R} 1} \otimes \mathbf{C}_{\mathrm{R} 1}^{-1} \mathbf{n}^{1}+\mathbf{F}_{\mathrm{R} 1}^{-T} \mathbf{n}^{1} \otimes \mathbf{C}_{\mathrm{R} 1}^{-1} \mathbf{E}_{\mathrm{R} 1}\right)\right] \mathbf{n}^{0}=\mathbf{0} .
\end{aligned}
$$

By taking the inner product of both members of Eq. (22) with $\mathbf{F m}^{0}$, all terms involving the pressures vanish, leading to the following equation for $\alpha$

$$
A(\alpha, \beta(\alpha)) \alpha+B(\alpha, \beta(\alpha))=0,
$$

in which 


$$
\begin{aligned}
A(\alpha, \beta(\alpha))= & {\left[c^{\mathrm{R} 1} \mu_{\mathrm{sh}}+c^{\mathrm{sh}} \mu_{V}-c^{\mathrm{sh}}\left(\mu_{V}-\mu_{R}\right)\left(\mathbf{n}^{0} \cdot \mathbf{n}^{1}\right)^{2}\right] } \\
& \times\left|\mathbf{F} \mathbf{m}^{0}\right|^{2} \\
& +\left\{\frac { c ^ { \mathrm { sh } } \mathbf { m } ^ { 0 } \cdot \mathbf { n } ^ { 1 } } { H ( \alpha ) } \left[2\left(\epsilon_{V}-\epsilon_{R}\right) T(\alpha, \beta(\alpha))\right.\right. \\
& \times \mathbf{E} \cdot \mathbf{m}^{0}-\frac{\mathbf{m}^{0} \cdot \mathbf{n}^{1}}{H(\alpha)}\left(\left(\mu_{V}-\mu_{R}\right)+\left(\epsilon_{V}-\epsilon_{R}\right)\right. \\
& \left.\left.\left.T(\alpha, \beta(\alpha))^{2}\right)\right]-\left(\mathbf{E} \cdot \mathbf{m}^{0}\right)^{2}\left(c^{\mathrm{R} 1} \epsilon_{\mathrm{sh}}+c^{\mathrm{sh}} \epsilon_{V}\right)\right\} \\
& \times\left|\mathbf{F}^{-T} \mathbf{n}^{0}\right|^{2},
\end{aligned}
$$

and

$$
\begin{aligned}
B(\alpha, \beta(\alpha))= & \left(\mu_{\mathrm{sh}}-\mu_{V}\right) \mathbf{C} \cdot\left(\mathbf{n}^{0} \otimes \mathbf{m}^{0}\right) \\
& +\left(\mu_{V}-\mu_{R}\right) \mathbf{n}^{0} \cdot \mathbf{n}^{1} \mathbf{C} \cdot\left(\mathbf{m}^{0} \otimes \mathbf{n}^{1}\right) \\
& +\left[\left(\epsilon_{\mathrm{sh}}-\epsilon_{V}\right) \mathbf{E} \cdot \mathbf{m}^{0}+\frac{\epsilon_{V}-\epsilon_{R}}{H(\alpha)} T(\alpha, \beta(\alpha)) \mathbf{m}^{0} \cdot \mathbf{n}^{1}\right] \mathbf{C}^{-1} \cdot\left(\mathbf{E} \otimes \mathbf{n}^{0}\right) \\
& +\beta\left[\left(c^{\mathrm{R} 1} \epsilon_{\mathrm{sh}}+c^{\mathrm{sh}} \epsilon_{V}\right) \mathbf{E} \cdot \mathbf{m}^{0}-\frac{c^{\mathrm{sh}}\left(\epsilon_{V}-\epsilon_{R}\right)}{H(\alpha)} T(\alpha, \beta(\alpha)) \mathbf{m}^{0} \cdot \mathbf{n}^{1}\right] \\
& \times\left|\mathbf{F}^{-T} \mathbf{n}^{0}\right|^{2}+\frac{\mathbf{C}^{-1} \cdot\left(\mathbf{n}^{0} \otimes \mathbf{n}^{1}\right)}{H(\alpha)}\left\{\left(\epsilon_{V}-\epsilon_{R}\right) T(\alpha, \beta(\alpha)) \mathbf{E} \cdot \mathbf{m}^{0}\right. \\
& \left.-\frac{\left[\mu_{V}-\mu_{R}+\left(\epsilon_{V}-\epsilon_{R}\right) T(\alpha, \beta(\alpha))^{2}\right] \mathbf{m}^{0} \cdot \mathbf{n}^{1}}{H(\alpha)}\right\}
\end{aligned}
$$

where

$$
\begin{aligned}
T(\alpha, \beta(\alpha))= & \mathbf{C}^{-1} \cdot\left(\mathbf{E} \otimes \mathbf{n}^{1}\right)+\alpha c^{\mathrm{sh}} \mathbf{m}^{0} \cdot \mathbf{n}^{1} \mathbf{C}^{-1} \cdot\left(\mathbf{E} \otimes \mathbf{n}^{0}\right) \\
& +c^{\mathrm{sh}}\left(\alpha \mathbf{E} \cdot \mathbf{m}^{0}-\beta\right) \mathbf{C}^{-1} \cdot\left(\mathbf{n}^{0} \otimes \mathbf{n}^{1}\right) \\
& +\alpha\left(c^{\mathrm{sh}}\right)^{2} \mathbf{m}^{0} \cdot \mathbf{n}^{1}\left(\alpha \mathbf{E} \cdot \mathbf{m}^{0}-\beta\right)\left|\mathbf{F}^{-T} \mathbf{n}^{0}\right|^{2}
\end{aligned}
$$

Equations (19) and (23) constitute the main analytical result of this investigation. We note that the technique leading to such a result, allowing one to skip the computation of the mesoscopic pressures to determine the macroscopic response, is not limited to the adopted neo-Hookean hyperelasticity (specified in Sect. 3). In fact, it just makes use of the choice of the deformation gradient as primal kinematic variable for the finite deformation framework.

Equation (23) for $\alpha$ can be coupled to the macroscopic boundary conditions (1b) and (1c) to compute the actuation stretch $\lambda$ and the amount of shear $\xi$. As demonstrated in Sect. 5, this procedure is computationally convenient as it allows one to avoid the evaluation of the mesoscopic pressures $p_{k}$. We remind that, as explained in Sect. 2, in our homogenisation algorithm, after the computation of $\alpha, \lambda$, and $\xi$, the pressure $p$ is evaluated through the analytical expression obtained by imposing Eq. (1a).

Now, we provide the relations to obtain the remaining mesoscopic fields. First of all, $\beta$ can be computed from Eq. (19). Second, the mesoscopic stress state requires the evaluation of $p_{\mathrm{R} 1}-p_{\mathrm{sh}}$, that can be obtained from the inner product of both members of Eq. (22) with $\mathbf{F}^{-T} \mathbf{n}^{0}$, that yields

$$
\begin{aligned}
p_{\mathrm{R} 1}-p_{\mathrm{sh}}= & \left|\mathbf{F}^{-T} \mathbf{n}^{0}\right|^{-2}\left\{\mu_{V}-\mu_{\mathrm{sh}}-\epsilon_{\mathrm{sh}}\left[\mathbf{C}^{-1} \cdot\left(\mathbf{E} \otimes \mathbf{n}^{0}\right)\right.\right. \\
& \left.+c^{\mathrm{R} 1}\left(\beta-\alpha \mathbf{E} \cdot \mathbf{m}^{0}\right)\left|\mathbf{F}^{-T} \mathbf{n}^{0}\right|^{2}\right]^{2} \\
& +\epsilon_{V}\left[\mathbf{C}^{-1} \cdot\left(\mathbf{E} \otimes \mathbf{n}^{0}\right)\right. \\
& \left.+c^{\mathrm{sh}}\left(\alpha \mathbf{E} \cdot \mathbf{m}^{0}-\beta\right)\left|\mathbf{F}^{-T} \mathbf{n}^{0}\right|^{2}\right]^{2} \\
& -\left(\mu_{V}-\mu_{R}\right)\left(\mathbf{n}^{0} \cdot \mathbf{n}^{1}\right)^{2} \\
& +\frac{\mu_{V}-\mu_{R}+\left(\epsilon_{V}-\epsilon_{R}\right) T(\alpha, \beta(\alpha))^{2}}{H(\alpha)^{2}} \\
& \times\left[\mathbf{C}^{-1} \cdot\left(\mathbf{n}^{0} \otimes \mathbf{n}^{1}\right)+\alpha c^{\mathrm{sh}} \mathbf{m}^{0} \cdot \mathbf{n}^{1}\left|\mathbf{F}^{-T} \mathbf{n}^{0}\right|^{2}\right]^{2} \\
& -2 T(\alpha, \beta(\alpha)) L(\alpha)\left[\mathbf{C}^{-1} \cdot\left(\mathbf{E} \otimes \mathbf{n}^{0}\right)\right. \\
& \left.\left.+c^{\mathrm{sh}}\left(\alpha \mathbf{E} \cdot \mathbf{m}^{0}-\beta\right)\left|\mathbf{F}^{-T} \mathbf{n}^{0}\right|^{2}\right]\right\} .
\end{aligned}
$$

Third, $\bar{\beta}$, defining the mesoscopic electric displacements through Eqs. (6), can be then obtained by directly employing Eqs. (17).

It is worth mentioning that, by assuming $\epsilon_{R}=\epsilon_{V}$ and $\mu_{R}=\mu_{V}$ in Eq. (13), such as the rank-one phase becomes microscopically homogeneous, the foregoing procedure particularises to that proposed by Gei et al. [4] for a rank-one composite, in turn numerically leading to the results analytically achievable through the potential (13).

\section{Application to optimised microstructures for rank-two laminates: validation and new layouts}

We now apply the procedure developed in Sect. 4 to actuators whose microscopic properties are the same as those adopted in $[2,5]$ to facilitate the comparison. In particular, for the matrix we adopt $\mu_{b}=10 \mathrm{MPa}$ and $\epsilon_{b}=10 \epsilon_{0}$, where $\epsilon_{0}$ is the permittivity of the vacuum, i.e. $\epsilon_{0}=8.85 \times 10^{-12} \mathrm{~F} / \mathrm{m}$. The numerical studies here illustrated are performed by implementing the standard and novel homogenisation procedures in the 
Table 1 Plane-strain rank-two configurations ('Tree a') optimised for maximum stretch $\left(\lambda_{\max }\right)$ for different contrast on shear modulus and permittivity at $E_{2}=100 \mathrm{MV} / \mathrm{m}$ : comparison with literature results [5]

\begin{tabular}{llllllll}
\hline$\frac{\mu_{a}}{\mu_{b}}=\frac{\epsilon_{a}}{\epsilon_{b}}$ & $c_{\mathrm{R} 1}$ & $c_{b}^{\mathrm{R} 1}$ & $\theta\left[{ }^{\circ}\right]$ & $\theta_{\mathrm{R} 1}\left[^{\circ}\right]$ & $\lambda_{\max }$ & $\xi$ & Gain w.r.t. homogeneous \\
\hline 1 & 0 & - & - & - & 1.0234 & 0 & - \\
10 & 0.831 & 0.468 & 20.1 & 62.1 & 1.0271 & 0.0013 & 1.16 \\
100 & 0.963 & 0.489 & 12.3 & 65.1 & 1.1241 & 0.1181 & 5.30 \\
1000 & 0.990 & 0.493 & -20.0 & 70.0 & 2.4789 & 0.2485 & 63.20 \\
\hline
\end{tabular}

The first row reports the homogeneous case

commercial software 'Mathematica' ver. 11.0 (Wolfram Research, Inc.).

First, we perform a comparative investigation by considering the layout 'Tree a', thoroughly analysed by Gei and Mutasa [5] for phase contrast up to 1000 on both the shear modulus, $\mu_{a} / \mu_{b}$, and the permittivity, $\epsilon_{a} / \epsilon_{b}$. We validate our results by assuming the configurations reported in Table 3 of $[5],{ }^{2}$ therein optimised with respect to the stretch $\lambda$ for applied macroscopic electric field $E_{2}=100 \mathrm{MV} / \mathrm{m}$. Table 1 reports our results, which are in excellent agreement with those published in [5], both in terms of stretch and amount of shear. In fact, the relative error never exceeds $10^{-6}$. The last column of Table 1 expresses the gain in longitudinal strain with respect to the homogeneous case, whose stretch is reported in the first row.

Second, we focus on the 'Tree b' layout, for which the only available study is within the small strains and rotations framework [2]. Hence, for this layout, by resorting to the routine 'FindMaximum' of 'Mathematica', we look for new optimal configurations to maximise $\lambda$ at the applied nominal electric field $E_{2}=100 \mathrm{MV} / \mathrm{m}$, as for the 'Tree a' case. To this end, we remark that, because of the problem nonlinearity, one has to carefully inspect the solutions for the mesoand micro-structural parameters to ensure that they correspond to achievable electro-mechanical fields. The optimisation results are reported in Table 2. They specifically refer to increasing shear modulus and permittivity ratios, $\mu_{a} / \mu_{b}$ and $\epsilon_{a} / \epsilon_{b}$, from 1 (homogeneous case) to 20 . As summarised by the data in the last column of Table 2, these analyses allow us to establish that the performance of the 'Tree b' rank-two

\footnotetext{
${ }^{2}$ In [5], the volume fraction of phase $b$ in the core corresponds to the ratio $c_{b}^{\mathrm{R} 1} / c_{\mathrm{R} 1}$.
}

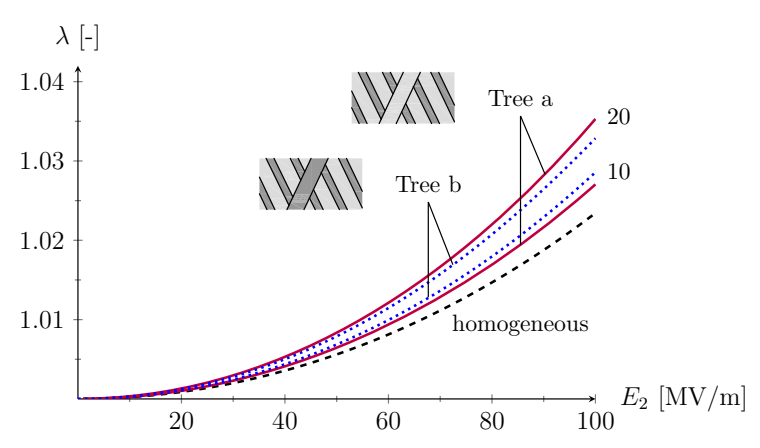

Fig. 2 Longitudinal stretch $\lambda$ for optimal rank-two devices ('Tree a' and 'Tree b') for $\mu_{a} / \mu_{b}=\epsilon_{a} / \epsilon_{b}=10$ and $\mu_{a} / \mu_{b}=$ $\epsilon_{a} / \epsilon_{b}=20$ calculated in plane-strain at increasing applied nominal electric field $E_{2}$

actuator improves with respect to the homogeneous case, analogously to the 'Tree a' layout.

However, as highlighted by the $\lambda-E_{2}$ curves plotted in Fig. 2, for a composite with $\mu_{a} / \mu_{b}=\epsilon_{a} / \epsilon_{b}=10$, the 'Tree b' layout achieves a slightly higher stretch, whereas the improvement in the actuation response exhibits opposite trend when the phase contrast is set to 20 , demonstrating the existence of a transition between the two behaviours in the interval between the two analysed values of the contrast. Moreover, the optimal 'Tree b' microstructures are obtained by decreasing both the volume fraction of the core and the angle of the core-shell interface $\theta$ with increasing phase contrast. The foregoing observations are in agreement with the findings reported in [2] and show that both layouts should be considered in the optimisation of actuators based on rank-two laminates. Our nonlinear results, better suited than those obtained in the linear regime, confirm the importance of carefully considering the composite conceived as islands of stiff reinforcements in a soft matrix (i.e., the intuitive 'Tree a' 
Table 2 Plane-strain rank-two configurations ('Tree b') optimised for maximum stretch $\left(\lambda_{\max }\right)$ for different contrast on shear modulus and permittivity at $E_{2}=100 \mathrm{MV} / \mathrm{m}$

\begin{tabular}{llllllll}
\hline$\frac{\mu_{a}}{\mu_{b}}=\frac{\epsilon_{a}}{\epsilon_{b}}$ & $c_{\mathrm{R} 1}$ & $c_{b}^{\mathrm{R} 1}$ & $\theta\left[{ }^{\circ}\right]$ & $\theta_{\mathrm{R} 1}\left[^{\circ}\right]$ & $\lambda_{\max }$ & $\xi$ & Gain w.r.t. homogeneous \\
\hline 1 & 0 & - & - & - & 1.0234 & 0 & - \\
5 & 0.600 & 0.450 & 54.4 & -57.9 & 1.0258 & -0.0017 & 1.10 \\
10 & 0.565 & 0.428 & 50.9 & -58.6 & 1.0286 & -0.0045 & 1.22 \\
15 & 0.526 & 0.405 & 48.5 & -59.9 & 1.0309 & -0.0075 & 1.32 \\
20 & 0.394 & 0.318 & 45.1 & -64.5 & 1.0329 & -0.0131 & 1.41 \\
\hline
\end{tabular}

The first row reports the homogeneous case. Some of the corresponding $\lambda-E_{2}$ curves are plotted in Fig. 2

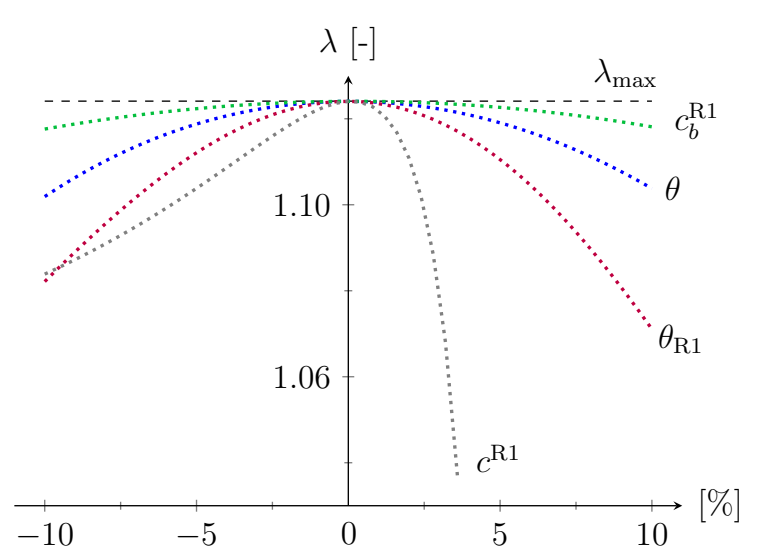

Fig. 3 Longitudinal stretch $\lambda$ for 'Tree a' layout with $\mu_{a} / \mu_{b}=$ $\epsilon_{a} / \epsilon_{b}=100$ as a function of the four meso- and micro-structural parameters $\theta, \theta_{\mathrm{R} 1}, c^{\mathrm{R} 1}$, and $c_{b}^{\mathrm{R} 1}$. The abscissa domain is $[-0.1 \gamma, 0.1 \gamma]$ where $\gamma$ is the maximum value achievable by the considered parameter. The maximum value of $c^{\mathrm{R} 1}$ is $3.7 \%$ that corresponds to $c^{\mathrm{R} 1}=1$

arrangement) as the only way to improve the response of an electro-elastic device and show that islands of soft domains hierarchically embedded in a relatively stiff matrix can reach even better actuation stretches.

To gain insight on the sensitivity of $\lambda_{\max }$ to changes of meso- and micro-structural parameters, we report in Figs. 3 and 4 the actuation stretch as a function of $\theta$, $\theta_{\mathrm{R} 1}, c^{\mathrm{R} 1}$, and $c_{b}^{\mathrm{R} 1}$. Specifically, we take single variations of such parameters about the optimal configuration within a range of $\pm 10 \%$ of their maximum possible values. In particular, Fig. 3 refers to the 'Tree a' layout with contrast $\mu_{a} / \mu_{b}=\epsilon_{a} / \epsilon_{b}=100$, while Fig. 4 is concerned with the 'Tree b' laminate with $\mu_{a} / \mu_{b}=\epsilon_{a} / \epsilon_{b}=20$.

For the 'Tree a' composite, the stretch is clearly more sensitive to variations of the core volume

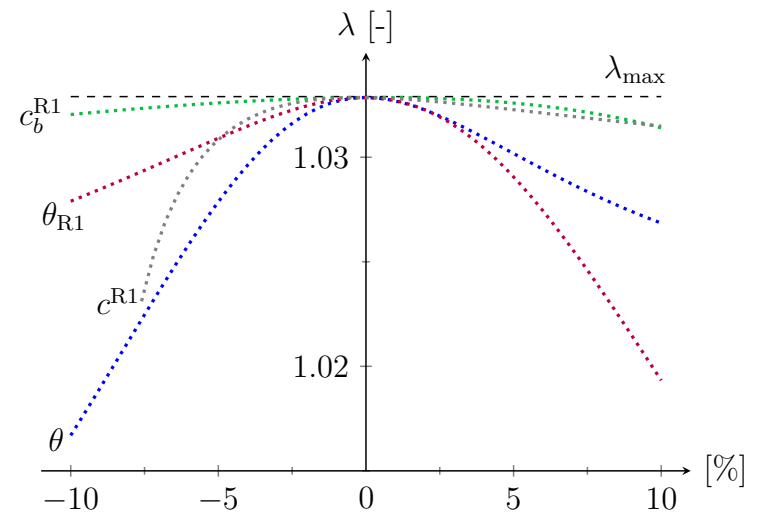

Fig. 4 Longitudinal stretch $\lambda$ for 'Tree b' layout with $\mu_{a} / \mu_{b}=$ $\epsilon_{a} / \epsilon_{b}=20$ as a function of the four meso- and micro-structural parameters $\theta, \theta_{\mathrm{R} 1}, c^{\mathrm{R} 1}$, and $c_{b}^{\mathrm{R} 1}$. The abscissa domain is $[-0.1 \gamma, 0.1 \gamma]$ where $\gamma$ is the maximum value achievable by the considered parameter. The minimum value of $c^{\mathrm{R} 1}$ is limited by $c_{b}^{\mathrm{R} 1}=0.318$ and corresponds to $-7.6 \%$

fraction and of the microscopic interface orientation $\theta_{\mathrm{R} 1}$, whereas for the 'Tree b' layout, the two angles $\theta$ and $\theta_{\mathrm{R} 1}$ play the most important role, at least in the neighbourhood of the optimal configuration.

Finally, to estimate the effectiveness of the new homogenisation procedure, the computational time required by the software to reach a convergent solution is evaluated through the built-in function 'Timing'. For different sets of applied parameters, it takes an average of about $0.45 \mathrm{~s}$ to converge with the new procedure developed in Sect. 4. By contrast, under the same conditions, about $4.09 \mathrm{~s}$ are needed in average to reach convergence with the homogenisation procedure so far adopted in literature, also requiring the computation of the mesoscopic pressures. This proves the superior efficiency of the homogenisation procedure here proposed, which is expected to be more robust 
and reduces the computational cost of about nine times.

\section{Concluding remarks}

Soft electro-elastic hierarchical laminated composites exhibit great potential for the realisation of voltagedriven devices with enhanced actuation properties. In particular, rank-two laminates represent a good compromise between the complexity associated with manufacturing and the actuation performance that hierarchical materials may provide.

In this paper, we have focused on nonlinear electroelastic problem for two rank-two composite layouts whose phases obey a neo-Hookean mechanical response augmented with an ideal dielectric response. For both layouts, we have shown how to simplify at most the system of solving equations. Our proposal relies on analytical treatments of the original set of equations directly formulated on accounting for the electro-mechanical continuity relationships at the shell-core interface and macroscopic external boundary conditions. We remark that the proposed analytical technique would apply also to study electro-elastic laminates whose phases obey other relevant hyperelastic laws, as, for instance, that proposed by Gent [21].

We have demonstrated that the proposed reduced set of solving equations is very efficient when attempting to find a numerical solution, whereby the required computational time is about one order of magnitude lower than that needed to solve the whole, untreated system.

As an additional outcome of this research, we have obtained, under plane-strain conditions and for the case of equal contrast between phase shear moduli and permittivities, new optimal configurations in terms of longitudinal actuation in our nonlinear framework for the rank-two layout where soft inclusions are embedded in a stiffer matrix, here referred to as 'Tree b'. We show that this arrangement can reach higher actuation stretches with respect to those achieved by the 'Tree a' configurations for relatively low contrasts.

We have finally assessed, for both types of composite, the sensitivity of the actuation stretch to perturbations of the meso- and micro-structural parameters, by varying them about the optimal configurations. We have observed a remarkable dependence of the results on the adopted layout (either 'Tree a' or 'Tree b'), also in terms of the most influencing parameter. However, the microscopic interface orientation (characterising the nested rankone phase) always plays a relevant role.

Future investigations should focus on developing a semi-analytical homogenisation procedure, analogous to that here proposed, to optimise the meso- and micro-structures of the rank-two dielectric composites under charge-controlled actuation. We expect such models to aid the design and development of optimal hierarchical dielectric composite actuators.

Acknowledgements L.B. acknowledges the support of the Italian Ministry of Education, University, and Research (MIUR). M.G. gratefully acknowledges the support of Royal Academy of Engineering, Grant No. DVF1718 $\backslash 8 \backslash 61$.

\section{Compliance with ethical standards}

Conflict of interest The Authors declare that they have no conflict of interest.

Open Access This article is distributed under the terms of the Creative Commons Attribution 4.0 International License (http:// creativecommons.org/licenses/by/4.0/), which permits unrestricted use, distribution, and reproduction in any medium, provided you give appropriate credit to the original author(s) and the source, provide a link to the Creative Commons license, and indicate if changes were made.

\section{Appendix}

When the deformation along $x_{3}$ is unconstrained, the number of independent macroscopic stretches increases to two. In particular, we select those associated with axes $x_{1}$ and $x_{2}$, indicated now as $\lambda_{1}$ and $\lambda_{2}$, respectively, so that in this case the deformation gradient takes the form

$$
\mathbf{F}=\left[\begin{array}{ccc}
\lambda_{1} & \xi \lambda_{2} & 0 \\
0 & \lambda_{2} & 0 \\
0 & 0 & 1 /\left(\lambda_{1} \lambda_{2}\right)
\end{array}\right]
$$

The lowest-term system for the solution of the boundary-value problem involves now variables

$$
\alpha, \lambda_{1}, \lambda_{2}, \xi \text {. }
$$

Their evaluation requires the use of the macroscopic boundary condition 


$$
S_{33}=0
$$

in addition to the already introduced conditions (23), (1b), and (1c).

The determination of the variables in Eq. (28) then allows one to compute all the remaining quantities by precisely following the procedure proposed in Sect. 4.

In this regard, on the one hand, our numerical experiments carried out on configurations analogous to those studied in Tables 1 and 2 confirm the computational superiority of the proposed procedure. On the other hand, they clearly demonstrate that the stretch along the $x_{3}$ direction does not play a relevant role in the electro-mechanics of the laminated composite structures here of interest, for which the planestrain assumption is then totally appropriate. However, the solution of the more general boundary-value problem could be relevant for hierarchical composites whose shear moduli ratio differs from that of dielectric permittivities.

\section{References}

1. deBotton G, Tevet-Deree L, Socolsky E (2007) Electroactive heterogeneous polymers: analysis and applications to laminated composites. Mech Adv Mater Struct 14:13-22

2. Tian L, Tevet-Deree L, deBotton G, Bhattacharya K (2012) Dielectric elastomer composites. J Mech Phys Solids 60:181-198

3. Rudykh S, Lewinstein A, Uner G, deBotton G (2013) Analysis of microstructural induced enhancement of electromechanical coupling in soft dielectrics. Appl Phys Lett 102:151905

4. Gei M, Springhetti R, Bortot E (2013) Performance of soft dielectric laminated composites. Smart Mater Struct 22:104014

5. Gei M, Mutasa K (2018) Optimisation of hierarchical dielectric elastomer laminated composites. Int J Nonlinear Mech 106:266-273

6. Bertoldi K, Gei M (2011) Instability in multilayered soft dielectrics. J Mech Phys Solids 59:18-42
7. Rudykh S, deBotton G (2011) Stability of anisotropic electroactive polymers with application to layered media. Z Angew Math Phys 62:1131-1142

8. Rudykh S, Bhattacharya K, deBotton G (2014) Multiscale instabilities in soft heterogeneous dielectric elastomers. Proc R Soc Lond A 470:20130618

9. Goshkoderia A, Rudykh S (2017) Electromechanical macroscopic instabilities in soft dielectric elastomer composites with periodic microstructures. Eur J Mech A Solids 65:243-256

10. Siboni M, Castañeda PP (2014) Fiber-constrained, dielectric-elastomer composites: finite-strain response and stability analysis. J Mech Phys Solids 68:211-238

11. Gei M, Springhetti R, Colonnelli S (2014) The role of electrostriction on the stability of dielectric elastomer actuators. Int J Solids Struct 51:848-860

12. Spinelli S, Lopez-Pamies O (2015) Some simple explicit results for the elastic dielectric properties and stability of layered composites. Int J Eng Sci 88:15-28

13. Polukhov E, Vallicotti D, Keip M-A (2018) Computational stability analysis of periodic electroactive polymer composites across scales. Comput Method Appl Mech Eng 337:195-197

14. Pelrine R, Kornbluh R, Joseph J (1998) Electrostriction of polymer dielectrics with compliant electrodes as a means of actuation. Sens Actuators A Phys 64:77-85

15. Keplinger C, Kaltenbrunner M, Arnold N, Bauer S (2010) Rontgen's electrode-free elastomer actuators without electromechanical pull-in instability. Proc Natl Acad Sci USA 107:4505-4510

16. Hill R (1963) Elastic properties of reinforced solids: some theoretical principles. J Mech Phys Solids 11:357-372

17. Hill R (1972) On constitutive macro-variables for heterogeneous solids at finite strain. Proc R Soc Lond A 326:131-147

18. Ogden R (1974) On the overall moduli of non-linear elastic composite materials. J Mech Phys Solids 22:541-553

19. deBotton G (2005) Transversely isotropic sequentially laminated composites in finite elasticity. J Mech Phys Solids 53:1334-1361

20. Dorfmann A, Ogden R (2005) Nonlinear electroelasticity. Acta Mech 174:167-183

21. Gent AN (1996) A new constitutive relation for rubber. Rubber Chem Technol 69(1):59-61

Publisher's Note Springer Nature remains neutral with regard to jurisdictional claims in published maps and institutional affiliations. 